\title{
The prevalence and determinants of vitamin $D$ deficiency in older Irish adults: Data from The Irish Longitudinal Study on Ageing (TILDA)
}

\author{
E. Laird ${ }^{1}$, A.M. O’Halloran ${ }^{1}$, D. Carey ${ }^{1}$, M. Healy ${ }^{2}$, D. O'Connor ${ }^{1}$, P. Moore ${ }^{1}$, T. Shannon ${ }^{2}$, \\ A.M. Molloy and R.A. Kenny, ${ }^{1,3}$ \\ ${ }^{1}$ The Irish Longitudinal Study on Ageing, Trinity College Dublin, Ireland, \\ ${ }^{2}$ The Department of Biochemistry \& Clinical Pathology, St James's Hospital, Ireland, \\ ${ }^{3}$ School of Medicine, Trinity College Dublin, Ireland and \\ ${ }^{4}$ Mercer's Institute for Successful Ageing, St. James's Hospital, Dublin, Ireland.
}

Older adults (>50 yrs) are heavily dependent on sunshine exposure to contribute to overall vitamin D status ${ }^{(1)}$. However, unlike the rest of the population, the ability for older adults to synthesize vitamin D is significantly reduced ${ }^{1}$. This coupled with age-related changes in metabolism such as increased food malabsoprtion, and dietary patterns characterized by a decrease in food quality, quantity and variety ${ }^{(2)}$ leaves the older population at increased risk of vitamin D deficiency. However, few data are available examining the in-depth health, lifestyle, geographic and socio-economic determinants of vitamin D status exclusively in older adults.

The plasma concentration of 25-hydroxyvitamin D $(25(\mathrm{OH}) \mathrm{D})$ was measured by LC-MS/MS in 5,356 community dwelling older Irish adults (aged 50-98 yrs) from The Irish Longitudinal Study on Ageing (TILDA) cohort (Wave 1 data collection, 2009 to 2011), the largest nationally representative study of older adults in Ireland. Vitamin D containing dietary supplements and medications and detailed demographic, geographic and socio-economic factors were assessed by questionnaire. Proportions of vitamin D deficiency prevalence were generated by season sampled, geographic area (Figure 1) and by population characteristics. Linear regression was used to investigate the association between $25(\mathrm{OH}) \mathrm{D}$ concentration and demographic, geographic, health and lifestyle variables.

The prevalence of vitamin D deficiency $(25(\mathrm{OH}) \mathrm{D}<30 \mathrm{nmol} / \mathrm{L})$ was $13 \cdot 1 \%(95 \% \mathrm{CI}$ : 12.1-14.2) after seasonal adjustment. Deficiency status was more prevalent in non-supplement users, in winter months, in smokers, in obese adults, the physically inactive, those living alone and in the oldest old $(>80 \mathrm{yrs})$. The main predictors $(\mathrm{P}<0.05)$ of $25(\mathrm{OH}) \mathrm{D}$ concentration were supplement use (coefficient nmol/L: 27.2 (95\% CI: $15 \cdot 3$ to $39 \cdot 2)$ ), smoking $(-8.9(-12 \cdot 6$ to $-5 \cdot 2)$, summer season $(5.9(2.7$ to $9 \cdot 1)$ and obesity $(-4.0(-6.3$ to $-1 \cdot 7)$.
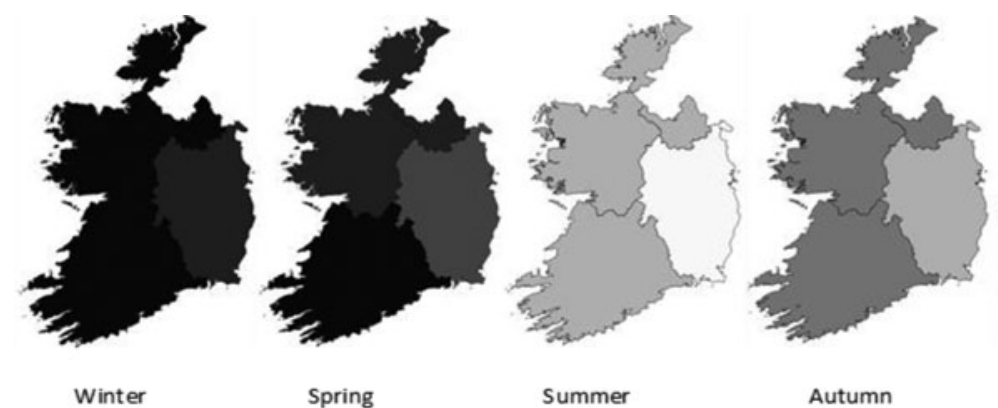

$\%<30 \mathrm{nmol} / \mathrm{L}$

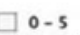

$5-10$

$5-10$
$10-15$

$10-15$
$15-20$

$15-20$
$20-25$

$25-30$

Fig. 1. Geographic and seasonal variation in the prevalence $(\%$ of population $)$ of $25(\mathrm{OH}) \mathrm{D}$ deficiency $(<30 \mathrm{nmol} / \mathrm{L})$ in older Irish adults.

Vitamin D deficiency is common among older Irish adults. These data indicate the need for targeted strategies within sections of the older population to improve vitamin D status and the need for increased awareness of the factors that can increase the risk of deficiency.

1. Laird E, Ward M, McSorely E, et al. (2010) Nutrients 7, 693-724.

2. Power SE, Jeffery IB, Ross RP et al. (2014) J Nutr Health Aging 18, 561-572. 